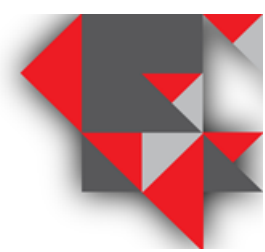

\title{
Sector y antigüedad como factores pertinentes en la perspectiva del empresario colombiano con relación a la capacidad de absorción
}

\author{
Victor Garcia-Valenzuela ${ }^{1}$; Paloma Gómez-Alvarez ${ }^{2}$ y Joanna Cota-Beltran ${ }^{3}$ \\ ${ }^{1}$ Instituto Tecnológico de Sonora, victor.garcia@potros.itson.edu.mx, Calle 5 de Febrero 818 Centro. CP \\ 85000 Cd. Obregón Sonora, 6444109000 \\ ${ }^{2}$ Instituto Tecnológico de Sonora, paloma.gomez111213@potros.itson.edu.mx, Calle 5 de Febrero 818 \\ Centro. CP 85000 Cd. Obregón Sonora, 6444109000 \\ ${ }^{3}$ Instituto Tecnológico de Sonora, Joanna.cota154359@potros.itson.edu.mx, Calle 5 de Febrero 818 Centro. \\ CP 85000 Cd. Obregón Sonora, 6444109000
}

Información del artículo revisado por pares

Fecha de aceptación: junio-2021

Fecha de publicación en línea: diciembre-2021

DOI: https://doi.org/10.29105/vtga7.2-7

\section{Resumen}

El entorno competitivo actual con el que se enfrentan las empresas cada vez es más complejo por lo que el constante cambio en las organizaciones se convierte en una necesidad. La capacidad dinámica de absorción del conocimiento juega un papel determinante para la competitividad en las empresas y para la generación de valor de las mismas, dicha capacidad consta de las fases de adquisición, asimilación, transformación y explotación del conocimiento con la finalidad de generar nuevo conocimiento y así incentivar el ecosistema de innovación organizacional. Por medio del método de análisis de varianza ANOVA de un factor se determinó con un nivel de confianza del $95 \%$ que el sector y la antigüedad de la empresa presentan variaciones estadísticamente significativas en la perspectiva de los empresarios con relación a las dimensiones de la capacidad dinámica de absorción del conocimiento.

Palabras clave: capacidad de absorción, capacidades dinámicas, gestión del conocimiento.

\begin{abstract}
Abstact
The current competitive environment that companies face is becoming more and more complex so that constant change in organizations becomes a necessity, the dynamic capacity to absorb knowledge plays a determining role for competitiveness in companies and for the generation of value thereof, said capacity consists of the phases of acquisition, assimilation, transformation and exploitation of knowledge in order to generate new knowledge and thus encourage the organizational innovation ecosystem. Through the one-factor ANOVA analysis of variance method, it was determined with a confidence level of $95 \%$ that the sector and the age of the company present statistically significant variations in the perspective of entrepreneurs in relation to the dimensions of dynamic capacity absorption of knowledge.
\end{abstract}

Keywords: absorption capacity, dynamic capacities, knowledge management.

JEL Codes: M1, M10, M12. 


\section{INTRODUCCIÓN}

En la actualidad las empresas se encuentran en un entorno inestable, los cambios que se viven ahora cada vez tienden a ser más radicales y más constantes. La supervivencia de las empresas tiende a propiciar este tipo de cambios en el mercado y en su entorno en un intento de sobrevivir a la mortalidad empresarial e incentivar su nivel de competitividad: entre mayor competente sea el entorno se generarán mayores cambios en busca de ser aun más competitivos, esto se determina como modelos complejos en economía según Perona (2005).

García et al. (2019b) indican que las economías poseen cierta complejidad en sus procesos productivos ya al ser desarrollados tienden a cambiar principalmente su entorno interno como externo debido a las nuevas condiciones de mejora en las que se encuentran las empresas al momento de desarrollar la complejidad en sus procesos. No obstante, para que una organización pueda generar complejidad en sus procesos para volverse más competitiva tiene que existir la aplicación de conocimiento complejo o especializado, sólo de esta forma se pueden generar ventajas competitivas que serán aprovechadas mediante estrategias empresariales por la organización.

Las ventajas competitivas sólo pueden ser sustentables si son generadas desde el capital humano de la organización. Para Nonaka y Takeuchi (1995) el talento humano es el recurso más importante de la empresa y es mediante el mismo donde se acumula, aplica y se genera conocimiento en las organizaciones. Por lo tanto, es el mismo talento humano que brinda las condiciones óptimas para que se genere mayor valor mediante un factor diferenciador que es traducido en ventajas competitivas.

La Capacidad Dinámica de Absorción del Conocimiento (CDAC) es una herramienta clave para la generación de valor en las organizaciones ya que gracias al desarrollo de esta capacidad dinámica las empresas pueden absorber el conocimiento externo, implementarlo y explotarlo con fines comerciales (Cohen y Levinthal, 1990). Esta capacidad está dividida en 4 fases o dimensiones y es en la última donde se genera nuevo conocimiento productivo elaborado desde una integración con el exterior y el ya existente en una organización por lo que se genera valor agregado para las organizaciones, creando así ventajas competitivas que posicionen mejor a la empresa en el mercado.

La mortalidad empresarial en Colombia juega un papel de gran incertidumbre para los empresarios ya que el elevado índice genera pragmatismo al momento de emprender o seguir emprendiendo. Las empresas colombianas, conforme Cajigas (2019), tienen una brecha de mortalidad de 5 años esenciales para determinar su perdurabilidad en el mercado. De cada 10 empresas solamente 3 logran sobrevivir y superar esta brecha de mortandad organizacional. Es por esto que la constante investigación en la generación de valor de las empresas es siempre un factor de importancia para la ciencia ya que el problema no sólo se ve en Colombia sino en un entorno internacional. García et al. (2019a) argumentan que la CDAC es un potencializador de valor que posibilita a las empresas a perdurar en el mercado.

Sánchez et al. (2017) atribuyen a las capacidades dinámicas como fuentes de generación de valor en las empresas y principalmente la de absorción de conocimiento ya que en ella se lleva acabo todo el proceso de gestión de conocimiento de las empresas. No obstante, aunque existen otras capacidades dinámicas que aumentan la competitividad de las organizaciones, la capacidad de absorción posee un elemento clave dentro de su proceso que es la innovación (Flor et al., 2018).

Antes de indagar a profundidad acerca de la CDAC es necesario conocer cómo el empresario está relacionado con la misma capacidad y que factores tienden a ser determinantes desde su perspectiva gerencial al momento de analizar la gestión de conocimiento de su organización. Anteriormente los autores García et al. 
(2019a) determinaron que los empresarios del sector industrial poseen sentido de pertinencia al momento de evaluar la CDAC en función a los factores de tamaño de la empresa y el sector al cual pertenecen mediante un análisis de medias (ANOVA) de un factor y concluyeron que el factor tamaño posee mayor importancia para los empresarios, descartando el factor de sector de los empresarios colombianos en su perspectiva por la CDAC.

López et al. (2016), mediante la misma metodología de ANOVA de un factor, determinan los factores con mayor importancia para los empresarios en cuanto a la sucesión familiar donde se produjo que al igual que los resultados de García et al. (2019) el factor tamaño es importante para la perspectiva del empresario y en este particular caso el sector al cual pertenecen también era de pertinencia para el empresario según sus resultados del modelo estadístico.

Castro et al. (2017) replican el modelo de ANOVA de un factor considerando los mismos factores tamaño y sector pero en función a la capacidad empresarial de los empresarios donde obtuvieron que de caso contrario con García et al. (2019a) el tamaño no es un factor de importancia y el factor de sector al cual pertenecen las empresas sí es un indicador estadísticamente significativo.

$\mathrm{Al}$ ser tan relevante la CDAC para las empresas aquí surge la pertinencia de realizar la presente investigación que pretende analizar bajo el mismo modelo estadístico de análisis de varianza ANOVA de un factor por determinar los factores de importancia de la perspectiva de los empresarios colombianos con respecto a las dimensiones de la CDAC, analizándolas en conjunto de los factores propuestos por los autores López et al. (2016), Castro et al. (2017), García et al. (2019a) y agregando los factores de número de empleados y antigüedad de las empresas para obtener un análisis más profundo de la perspectiva gerencial colombiana. Por lo que se plantea el siguiente objetivo de la investigación:

Encontrar la incidencia de impacto en cuanto a la pertinencia de cada uno de los factores en relación con las dimensiones de la capacidad dinámica de absorción del conocimiento de la perspectiva empresarial de Bucaramanga, Santander.

\section{MARCO TEÓRICO}

El término de la CDAC surge dentro de la microeconomía por Cohen y Levinthal (1990) la cual al definen como un proceso por el cual pasan las empresas al momento de encontrarse con conocimiento externo, dicho proceso consta de identificar el conocimiento externo para así lograr aprovecharlo e implementarlo en sus procesos productivos con fines comerciales, siendo esto la primera referencia conceptual de un esquema de aprendizaje en las organizaciones.

Posteriormente Zahra y George (2002) determinan la CDAC como un conglomerado de distintos procesos que conlleva la gestión de conocimiento en las empresas y lo estructuran en cuatro fases o dimensiones consecutivas: adquisición, asimilación, transformación y explotación.

La dimensión de adquisición es la primera de este proceso de la CDAC y consta en adquirir el conocimiento externo por parte de la empresa de tal forma que sea capaz de realizar la siguiente dimensión que es la de asimilación, esta se refiere a que la empresa sea capaz no solo de adquirirlo sino de entender el nuevo conocimiento adquirido de tal forma que pueda procesar la nueva información de manera correcta (Vega \& Schmutzler, 2017). La dimensión de transformación es esencial para la empresa es cuando el conocimiento ya se vuelve de explícito a tácito y es cuando la empresa una vez que lo asimila puede implementarlo en sus procesos productivos organizacionales, el cual en una integración con el conocimiento ya existente la empresa aplica ambos conocimientos; tras la aplicación de este conocimiento y la integración del anterior surge la cuarta y última dimensión la cual es la explotación del conocimiento que es cuando la empresa es capaz de generar nuevo conocimiento productivo con base en el adquirido anteriormente, es decir, surge la 
innovación en las empresas (Vega et al., 2018).

El aprovechar le conocimiento externo no solo convierte a la empresa más vanguardista a los cambios que suceden en el mercado sino que tambien incrementa la competitividad en la organización, la capacidad de absorción permite a las empresas incluirse en un esquema de gestión de conocimiento optimo para la organizacón según Tortoriello (2015).

Conforme Flatten et al. (2015) la capacidad de absorción del conocimiento esta totalmente relacionada con el talento humano de la organización, los directivos tienen gran influencia en esta capacidad ya que en ellos esta la gestión del conocimiento de su empresa; y más allá de la gestión, tambien tienen en sus responsabilidades el plan de acción (estrategia) a realizar una vez que se genera nuevo conocimiento, es por esto que el empresario, dueño o gerente tiene un papel determinande dentro de la CDAC.

De acuerdo con Pérez et al. (2019)

la CDAC fortalece la gestión del conocimiento en las empresas ya que esta capacidad no solo les permite a las empresas a identificar el conocimiento exterior al alcance de la empresa como área de oportunidad, sino también a llevar acabo un optimo aprendizaje del mismo donde la organización sea capaz de implementarlo con el objeto de que la empresa pueda generar nuevo conocimiento el cual incentivara la generación de valor de la empresa. En coincidencia con la teoría, aquellas empresas que sean capaces de desarrollar la CDAC, podrán establecer un entorno más competitivo ante el mercado mediante sus propuestas de mejora ya sea en sus procesos productivos, financieros $\mathrm{o}$ administrativos las cuales serán resultado de la última dimensión de la CDAC.

Valencia et al. (2016) mencionan que el capital humano es esencial para la absorción de conocimiento en las empresas, aunado a lo que dice Nonaka \& Takeuchi (1995) el capital intangible específicamente el talento humano es el más importante para generar valor y el medio para absorber conocimiento.

\section{METODOLOGÍA}

En relación con los objetivos de la investigación, esta posee un alcance descriptivo comparativo con un análisis de tipo cuantitativo por el tipo de información abstraída por el instrumento de recopilación de datos. La información fue recolectada en un tiempo único por lo que se denomina corte transversal y al no manipularse ninguna de las variables analizadas la investigación tiene un diseño no experimental. Dicho instrumento es una encuesta que consta de 16 items para medir cada una de las dimensiones de la CDAC y fue aplicado a los gerentes, dueños o puestos semejantes de las empresas encuestadas, la muestra no probabilística y a conveniencia consta de 90 empresas de Bucaramanga, Santander de los dos sectores más importantes del departamento colombiano según la Cámara de Comercio de Bucaramanga (2018). La muestra está divida por 45 empresas del sector servicio y 45 empresas del sector industrial, cada uno de los sectores esta subdividido por 5 giros diferentes que conforman cada sector, se encuestaron 9 empresas de cada giro de cada sector para evitar cualquier tipo de sesgo en la recolección de datos.

Los datos obtenidos por el instrumento se analizaron bajo una escala de Likert de 1 a 5 , donde 5 es el valor máximo correspondiente a la afirmación del item determinado y 1 es el valor mínimo correspondiente de la negación del item determinado. Anticipado a la aplicación del instrumento se realizó una prueba piloto para validar la confiabilidad del instrumento, posteriormente se hicieron las debidas adecuaciones al mismo para poder llevar a cabo el trabajo de campo.

La operacionalización de las variables es determinada por las dimensiones de la CDAC (adquisición, asimilación, transformación y explotación) como variables dependientes y como variables independientes 
o factores de comparación de media al sector, tamaño, numero de empelados y antigüedad de las empresas encuestadas, esto queda detallado en la Tabla 1.

Tabla 1

Operacionalización de las variables de estudio

\begin{tabular}{|c|c|c|c|c|c|}
\hline \multicolumn{5}{|c|}{ Variables independientes o factores } & Variable dependiente \\
\hline $\begin{array}{l}\text { Sector } \\
\text { Servicio }\end{array}$ & Industrial & Tamaño & No. Empleados & Antigüedad & $\begin{array}{l}\text { Capacidad dinámica de } \\
\text { absorción del conocimiento }\end{array}$ \\
\hline Aseguradora & Mueblería & Micro & $<10$ & $<1$ año & Adquisición \\
\hline Financiero & Confección & Pequeña & $>10 y<30$ & $>1$ año y $<5$ años & Asimilación \\
\hline Hotelero & Alimentos & Mediana & $>30 y<50$ & $>5$ años $y<10$ años & Transformación \\
\hline Inmobiliaria & Panadería & Grande & $>50 y<100$ & $>10$ años y $<15$ años & Explotación \\
\hline Salud & Calzado & & $\begin{array}{l}>100 y<250 \\
>250\end{array}$ & $\begin{array}{l}>15 \text { años y }<20 \text { años } \\
>20 \text { años }\end{array}$ & \\
\hline
\end{tabular}

Fuente: Elaboración propia.

La Tabla 1 especifica la detalladamente la operacionalización de cada variable así como sus dimensiones o características al momento de ser analizadas, las variables independientes $\mathrm{o}$ factores de tamaño y sector de las empresas son los utilizados y propuestos por López et al. (2016); Castro et al. (2017) y García et al. (2019) para medir la perspectiva del empresario, aparte de estos factores se agregan al estudio los años de antigüedad de las organizaciones y el numero de empleado de cada una de ellas, esto con la finalidad de lograr captar de una forma más completa la perspectiva del empresario al momento de persuadir en la CDAC. Las dimensiones de la variable dependiente son las mismas determinadas por Zahra \& George (2002) en su modelo de CDAC.

El método estadístico con el cual se analizan las variables es mediante un análisis estadístico de medias (ANOVA de un factor), mismo que lleva acabo una comparación de diferentes grupos en una variable con respecto a dos muestras independientes de la prueba $\mathrm{T}$ con necesariamente dos o más grupos, la sumatoria total de las distribuciones de las observaciones de las distancias al promedio general (varianza) en relación a la variable independiente determinado como factor corrobora la similitud de medias en los casos de las observaciones de la variable dependiente. La hipótesis del método estadístico es establecer si existe una similitud de medias en cada uno de los factores que conforman el modelo planteado por las variables independientes (sector, tamaño, antigüedad y número de empleados). En caso de aceptación de la hipótesis, esto quiere decir que la variable dependiente no tiene alteraciones en su comportamiento por lo cual su varianza no se ve modificada ante la presencia de los factores independientes categóricos. Esto se determina mediante el estadístico $\mathrm{F}$ de Fisher-Snedecor el cual establece que al ser menor o igual a 0.05 con un nivel de confianza del $95 \%$ el rechazo de la hipótesis de similitud existente en la comparación de medias. En el caso de la presente investigación, se realizan 4 pruebas de ANOVA de un factor donde se enfrenta cada una de las variables independientes con la variable dependiente (dimensiones CDAC) con la finalidad de determinar la inexistencia de similitud de medias en los promedios poblacionales para cada factor. Antes de aplicar el método estadístico se realizo un promedio ponderado para cada una de las dimensiones obtenido mediante la sumatoria total de cada item correspondiente a cada dimensión de la CDAC y dividiéndolo entre la cantidad de $n$ ítems por dimensión.

\section{RESULTADOS}

A continuación, se presentan los resultados de la aplicación del método estadístico con relación a las variables de estudio, en primera 
instancia se muestra en la Tabla 2 el ANOVA

tamaño con relación a las dimensiones de la de un factor correspondiente a la variable CDAC.

Tabla 2

ANOVA de un factor (tamaño)

\begin{tabular}{ccccccc}
\hline & & Suma cuadrado & gl & Media cuadrática & F & Sig. \\
\hline \multirow{4}{*}{ adquisición } & Inter-g & 1.564 & 3 & .521 & .620 & .604 \\
& Intra-g & 72.336 & 86 & .841 & & \\
& Total & 73.900 & 89 & & & \\
& Inter-g & 3.335 & 3 & 1.112 & 1.020 & .388 \\
asimilación & Intra-g & 93.697 & 86 & 1.090 & & \\
& Total & 97.032 & 89 & & & \\
& Inter-g & .356 & 3 & .119 & .138 & .937 \\
transformación & Intra-g & 74.188 & 86 & .863 & & \\
& Total & 74.544 & 89 & & & \\
& Inter-g & 1.612 & 3 & .537 & .475 & .701 \\
explotación & Intra-g & 97.301 & 86 & 1.131 & & \\
& Total & 98.914 & 89 & & & \\
\end{tabular}

La Tabla 2 muestra los resultados encontrados de la comparación de medias en cuanto las dimensiones de la capacidad dinámica de absorción del conocimiento y el factor tamaño, conforme a los resultados de la prueba de $\mathrm{T}$, el factor tamaño no es estadísticamente significativo para la

Tabla 3

ANOVA de un factor (Sector)

\begin{tabular}{|c|c|c|c|c|c|c|}
\hline & & Suma cuadrados & $\mathrm{gl}$ & Media cuadrática & $\mathrm{F}$ & Sig. \\
\hline & Inter-g. & 23.428 & 9 & 2.603 & 4.126 & .000 \\
\hline \multirow[t]{3}{*}{ adquisición } & Intra-g. & 50.472 & 80 & .631 & & \\
\hline & Total & 73.900 & 89 & & & \\
\hline & Inter-g. & 19.600 & 9 & 2.178 & 2.250 & .027 \\
\hline \multirow[t]{3}{*}{ asimilación } & Intra-g. & 77.432 & 80 & .968 & & \\
\hline & Total & 97.032 & 89 & & & \\
\hline & Inter-g. & 24.273 & 9 & 2.697 & 4.292 & .000 \\
\hline \multirow[t]{3}{*}{ transformación } & Intra-g. & 50.272 & 80 & .628 & & \\
\hline & Total & 74.544 & 89 & & & \\
\hline & Inter-g. & 27.704 & 9 & 3.078 & 3.458 & .001 \\
\hline \multirow[t]{2}{*}{ explotación } & Intra-g. & 71.210 & 80 & .890 & & \\
\hline & Total & 98.914 & 89 & & & \\
\hline \multicolumn{3}{|c|}{$\begin{array}{l}\text { La Tabla } 3 \text { muestra con un nivel de } \\
\text { nza del } 95 \% \text { que el factor como variable }\end{array}$} & \multicolumn{4}{|c|}{$\begin{array}{l}\text { sector indica que es estadísticament } \\
\text { significativo para la percepción del empresari }\end{array}$} \\
\hline $\begin{array}{l}\text { ependiente cate } \\
\text { erencia de medi } \\
\text { comparación de } \\
\text { ianza, el mode }\end{array}$ & $\begin{array}{l}\text { dependiente categórica sector si presenta } \\
\text { erencia de medias al momento de analizar }\end{array}$ & $\begin{array}{l}\text { si presenta } \\
\text { o de analizar } \\
\text { cuanto a su } \\
\text { con el factor }\end{array}$ & \multicolumn{4}{|c|}{$\begin{array}{l}\text { resultados del análisis de varianza del facto } \\
\text { número de empleados con relación a la } \\
\text { dimensiones de la CDAC. }\end{array}$} \\
\hline \multicolumn{3}{|c|}{$\begin{array}{l}\text { Tabla } 4 \\
\text { ANOVA de un factor (número de empleados) }\end{array}$} & & & & \\
\hline
\end{tabular}

percepción del empresario colombiano al momento de analizar la CDAC con un nivel de confianza del $95 \%$.

La Tabla 3 muestra los resultados correspondientes al ANOVA de un factor con respecto a la variable independiente sector. 


\begin{tabular}{|c|c|c|c|c|c|c|}
\hline & & Suma cuadrado & $\mathrm{gl}$ & Media cuadrática & $\mathrm{F}$ & Sig. \\
\hline \multirow{3}{*}{ adquisición } & Inter-g. & 16.287 & 5 & 3.257 & 4.749 & .001 \\
\hline & Intra-g. & 57.613 & 84 & .686 & & \\
\hline & Total & 73.900 & 89 & & & \\
\hline \multirow{3}{*}{ asimilación } & Inter-g. & 9.715 & 5 & 1.943 & 1.869 & .108 \\
\hline & Intra-g. & 87.317 & 84 & 1.039 & & \\
\hline & Total & 97.032 & 89 & & & \\
\hline \multirow{3}{*}{ transformación } & Inter-g. & 9.835 & 5 & 1.967 & 2.553 & .034 \\
\hline & Intra-g. & 64.710 & 84 & .770 & & \\
\hline & Total & 74.544 & 89 & & & \\
\hline \multirow{3}{*}{ explotación } & Inter-g. & 13.440 & 5 & 2.688 & 2.642 & .029 \\
\hline & Intra-g. & 85.474 & 84 & 1.018 & & \\
\hline & Total & 98.914 & 89 & & & \\
\hline
\end{tabular}

Los resultados de la Tabla 4 indican que bajo un nivel de confianza del $95 \%$ el factor número de empleados no es un factor estadísticamente determinante para la percepción del empresario al momento de considerar la dimensiones de la CDAC, por lo que existe una similitud de medias en cuanto

al comportamiento de las observaciones analizadas.

Por último, la Tabla 5 muestra los resultados correspondientes al análisis de ANOVA de un factor correspondiente a la variable independiente antigüedad de las empresas analizadas con relación a las dimensiones de la CDAC.

Tabla 5

ANOVA de un factor (Antigüedad)

\begin{tabular}{|c|c|c|c|c|c|c|}
\hline & & Suma cuadrado & $\mathrm{gl}$ & Media cuadrática & $\mathrm{F}$ & Sig. \\
\hline & Inter-g. & 16.835 & 5 & 3.367 & 4.956 & .001 \\
\hline \multirow[t]{3}{*}{ adquisición } & Intra-g. & 57.065 & 84 & .679 & & \\
\hline & Total & 73.900 & 89 & & & \\
\hline & Inter-g. & 23.623 & 5 & 4.725 & 5.406 & .000 \\
\hline \multirow[t]{3}{*}{ asimilación } & Intra-g. & 73.409 & 84 & .874 & & \\
\hline & Total & 97.032 & 89 & & & \\
\hline & Inter-g. & 17.576 & 5 & 3.515 & 5.183 & .000 \\
\hline \multirow[t]{3}{*}{ transformación } & Intra-g. & 56.968 & 84 & .678 & & \\
\hline & Total & 74.544 & 89 & & & \\
\hline & Inter-g. & 27.717 & 5 & 5.543 & 6.540 & .000 \\
\hline \multirow[t]{2}{*}{ explotación } & Intra-g. & 71.197 & 84 & .848 & & \\
\hline & Total & 98.914 & 89 & & & \\
\hline
\end{tabular}

Conforme a la Tabla 5 , los resultados indican con un nivel de confianza del $95 \%$ que el factor antiguiedad de las empresas si es un factor determinante para los empresarios al momento de analizar las dimensiones de la CDAC, lo que indica que no existe una similitud de medias en las distribuciones de las observaciones bajo el análisis de su varianza.
La capacidad dinámica de absorción del conocimiento de las empresas colombianas es una variable poco estudiada, es por eso que es importante analizar con detalle cada aspecto determinante de la misma. Los resultados encontrados con relación a los objetivos de la investigación indican que con un nivel de confianza del $95 \%$ dos factores de los cuatro analizados tienden a ser determinantes para la perspectiva del empresario al momento de considerar la gestión del conocimiento dentro de su organización.

\section{CONCLUSIONES}


Partiendo de la teoría, los factores que son determinantes al momento de considerar la capacidad dinámica de las empresas para absorber el conocimiento externo es el sector al cual pertenecer, esto independientemente al giro en especifico al cual se dediquen; y el factor de los años que tiene la empresa en el mercado activo en dicho sector. Estos dos factores son estadísticamente significativos al momento de analizar la gestión de conocimiento en las empresas, específicamente en el proceso de absorción de conocimiento externo para implementarlo en sus procesos productivos y generar nuevo conocimiento para su empresa mediante la innovación (Escalante y Balderrama, 2017).

Para el caso de los factores tamaño y número de empleados no se presenta alteración en la media promedio al momento de compararla mediante el análisis de varianza, lo cual indica que se acepta la hipótesis del modelo ANOVA al existir similitud en las distancias en las distribuciones observadas en el modelo, por lo que al momento de considerar la capacidad dinámica de absorción del conocimiento en las empresas en cuanto a la gestión de conocimiento dentro de la organización, el tamaño de la empresa y el número de empleados no es un factor determinante para el empresario, esto deduce que existen otros factores dentro de la perspectiva del empresario colombiano que ayudan considerar las dimensiones de la CDAC en su empresa.

El resultado de la investigación funge de gran utilidad en primera instancia a los empresarios al conocer estadísticamente y con un nivel considerable de confianza aquellos factores que pueden llegar a persuadir su perspectiva en al analizar la capacidad dinámica de absorción del conocimiento, de igual forma el gobierno del departamento santandereano tiene a su disposición las áreas de oportunidad para incentivar mediante programas públicos la gestión del conocimiento en las empresas; y por último la comunidad científica a generar nuevo y productivo conocimiento con fundamento estadístico sobre la perspectiva del empresario ante la capacidad dinámica de absorción de conocimiento, esto sirve como un constructo solido de antecedente para el estudio de las empresas colombianas y la gestión de conocimiento de las mismas.

\section{REFERENCIAS}

Cajigas, M. (2019). Propuesta de una Estrategia para Fortalecer la Capacidad Emprendedora en Colombia [Tesis de doctorado, Universidad de Granad]. Universidad de Granada. http://hdl.handle.net/10481/55752

Cámara de Comercio de Bucaramanga (2018) Producto interno Bruto Santander 2017: Actualidad económica. Observatorio de Competitividad. Julio 2018. Número 148. https://www.camaradirecta.com/temas/documentos\%20pdf/informes\%20de\%20actualidad/ 2018/PIB\%20Santander\%202017.pdf

Castro, J., Vásquez, M. y Ruiz, M. (2017) Variaciones en la valoración de los propietarios de empresas familiares en relación a los determinantes de la capacidad administrativa. Memorias del XXI Congreso Internacional de Investigación en Ciencias Administrativas 2017. ISBN: 978-607-8153-43-5 p. 1847-1862.

Cohen, W., M. \& Levinthal, D., A. (1990). Absorptive Capacity: A New Learning Perspective on Learning and Innovation. Administrative Science Quarterly,35(1), 128-152. https://doi.org/10.2307/2393553 
Escalante, J. C. C., \& Balderrama, J. I. L. (2017). Capacidad de absorción y competitividad en el cultivo de camarón del municipio de Ahome, Sinaloa. Estudios Sociales. Revista de alimentación contemporánea y desarrollo regional, 27(50). https://datos.ciad.mx/estudiosociales/index.php/es/article/view/475/482

Flatten, T., Adams, D. \& Brettel, M. (2015). Fostering absorptive capacity through leadership: A cross-cultural analysis. Journal of World Business, 50, 519-534. https://doi.org/10.1016/j.jwb.2014.08.010

Flor, ML, Cooper, S. \& Oltra, MJ (2018). Search for external knowledge, absorption capacity and radical innovation in high-tech companies. European Management Journal, 36 (2), 183-194.

García, V M., Meza, L. C., y Pedraza, F. (2018). Desarrollo de la capacidad dinámica de absorción de conocimiento entre empresas familiares y no familiares de Bucaramanga. Colombia. Revista Lebret, 10, 89-109 https://doi.org/10.15332/rl.v0i10.2199

García, V., López-Parra, M., Meza-Ariza, L., Bautista, F. (2019) Variación de la perspectiva empresarial colombiana en relación a las dimensiones de la capacidad dinámica de absorción del conocimiento. The Institute of Business and Finance Research. 12, (2). ISSN 1941-9589.

García, V., Ruiz, M., López-Parra, M. y Placencia, I. (2019) Diversidad y ubicuidad como determinantes de la complejidad económica de los municipios de Sonora. Emprendimiento e innovación empresarial. HESS S.A. DE C.V. Tomo 3, 585 - 624. ISBN: 978-607-9011-765.

López, M., Ruiz, M. y Meza, E. (2016). Variación en la percepción de los empresarios sobre la sucesión según el tamaño y giro de la empresa familiar. Ciencias Administrativas, Teoría y Praxis, 12(2) 267-283

Nonaka, I., \& Takeuchi, H. (1995). The knowledge-creating company: How Japanese companies create the dynamics of innovation. Oxford university press. ISBN 0-19-509269-4

Pérez Hernández, C. C., Moheno Mendoza, J., \& Salazar Hernández, B. C. (2019). Análisis estadístico de la capacidad de absorción en México y su influencia en la generación de conocimiento tecnológico. Innovar, 29(72), 41-58. http://www.scielo.org.co/pdf/inno/v29n72/0121-5051-inno-29-72-41.pdf

Perona, E. (2005). Ciencias de la complejidad: ¿La economía del siglo 21? Documento de Trabajo No. 22, Vol. 4. Departamento de Economía. http://portal.eco.unc.edu.ar/files/DeptoEconomiayFinanzas/SerieDocumdeTrab/DocN\%C2 \%BA22.pdf

Sánchez, E. O. P., Jaramillo, I. D. T., \& Sánchez, B. Y. H. (2017). Medición de la capacidad de absorción potencial en las empresas innovadoras de Colombia. Espacios, 38(26), 28-48.

Tortoriello, M. (2015). The social underpinnings of absorptive capacity: the moderating effects of structural holes on innovation generation based on external knowledge. Strategic Management Journal, 36(4), 586-597. http://dx.doi.org/10.1002/smj.2228 
Vega-Jurado, J. y Schmutzler, J. (2017). Determinantes del uso de fuentes externas de conocimiento en los procesos de innovación empresarial: la importancia de la dimensión regional. Espacios, $38(19), 23-41$.

Vega-Jurado, J., Polo-Otero, J., Cotes-Torres, M., \& Vega-Cárcamo, J. (2018). La base de conocimiento y su impacto en la capacidad de absorción de pymes de baja tecnología. Cuadernos De Administración, 30(55), $\quad 7 \quad$ - 35. https://doi.org/10.11144/Javeriana.cao30-55.bcica

Zahra, S. \& George, G. (2002). Absorptive capacity: A review, reconceptualization, and extension. $\begin{array}{llll}\text { Academy of } & \text { Management } & \text { Review, }\end{array}$ http://dx.doi.org/10.5465/amr.2002.6587995 